\title{
AGREGAÇÃO DE LATOSSOLO VERMELHO DISTRÓFICO TÍPICO RELACIONADA COM O MANEJ O NA REGIÃO DOS CERRADOS NO ESTADO DE MINAS GERAIS ${ }^{(\mathbf{1})}$
}

\author{
A. N. BEUTLER ${ }^{(2)}$, M. L. N.SILVA(3), N.CURI(3), M. M. FERREIRA(3), \\ I. A. PEREIRA FILHO(4) \& J . C. CRUZ(4)
}

\begin{abstract}
RESUMO
As conseqüências diretas do manejo inadequado do solo são a erosão, a redução da produtividade e a perda de sua sustentabilidade. Os indicadores da qualidade da estrutura do solo são importantes ferramentas para avaliar a sustentabilidade dos sistemas de manejo. Este estudo objetivou avaliar a agregação de um Latossolo Vermelho distrófico típico sob diferentes sistemas de manejo na região dos cerrados em Sete Lagoas (MG). Dentre os sistemas de manejo estudados, a semeadura direta foi o que apresentou a maior percentagem de agregados na classe $>2 \mathrm{~mm}$, menores nas classes $<2 \mathrm{~mm} \mathrm{e}<1 \mathrm{~mm}$ e maior diâmetro médio geométrico dos agregados na camada superficial $(0-5 \mathrm{~cm})$. A matéria orgânica apresentou correlação significativa $(P<0,01)$ e positiva com o índice de floculação $(r=0,89)$, diâmetro médio geométrico $(r=0,97)$, classe de agregados $>2 \mathrm{~mm}(r=0,92)$ e correlação negativa com classes de agregados $<2$ $(r=-0,92)$ e $<1 \mathrm{~mm}(r=-0,93)$. Esses aspectos ressaltam o efeito benéfico da semeadura direta, contribuindo para o manejo sustentado do solo.
\end{abstract}

Termos de indexação: semeadura direta, índices de agregação, estrutura.

(1) Parte da Tese de Mestrado do primeiro autor, apresentada ao Departamento de Ciência do Solo - DCS, Universidade Federal de Lavras - UFLA, curso de Pós-Graduação em agronomia. Trabal ho apresentado no XXVII Congresso Brasileiro de Ciência do Solo, Brasília, DF , 11 a 16 de julho de 1999. Recebido para publicação em outubro de 1999 e aprovado em setembro de 2000.

(2) Estudante do Curso de Pós-Graduação do Departamento de Ciência do Solo, Universidade Federal de Lavras - UFLA. Caixa Postal 37, CEP 37200-000 Lavras (MG). Bolsista da CAPES.

(3) Professor do Departamento de Ciência do Solo, Universidade Federal de Lavras - UFLA. Bolsista do CNPq.

(4) Pesquisador da Embrapa de Milho e Sorgo. Rod. MG 424, km 65, Caixa Postal 151, CEP 35701-970 Sete Lagoas (MG). 


\title{
SUMMARY: AGGREGATION OF TYPIC DYSTROPHIC RED LATOSOL (OXISOL) RELATED TO MANAGEMENT AT CERRADO REGION IN MINAS GERAIS, BRAZIL
}

\begin{abstract}
The direct consequences of inadequate soil management are erosion, reduction of productivity and loss of sustainability. Theindicators of soil structurequal ity arei mportant tools for evaluating sustainability of management systems. This study aimed to evaluatethe aggregation of typic dystrophic Red Latosol (Oxisol) under different management systems at cerrado regi on in Sete Lagoas, Minas Gerais, Brazil. Among the studied management systems, no-tillagepresented higher percentage of aggregates in the $>2 \mathrm{~mm}$ class, smaller percentage in the $<2 \mathrm{~mm}$ and $<1 \mathrm{~mm}$ classes, and larger geometric mean diameter of aggregates in thesuperficial layer $(0-5 \mathrm{~cm})$. Organic matter presented positiveand significant $(P<0.01)$ correlation with the flocculation index $(R=0.89)$, geometric mean diameter $(R=0.97),>2 \mathrm{~mm}$ aggregate class $(R=0.92)$, and negative correlation with $<2 \mathrm{~mm}$ $(R=-0.92)$ and $<1 \mathrm{~mm}(R=-0.93)$ aggregateclasses. Theseaspects emphasizethebeneficial effect of no-tillage, contributing for a sustainabl esoil management.
\end{abstract}

Index terms: no-tillage, aggregation indices, structure.

\section{NTRODUÇÃO}

A região dos cerrados ocupa $24 \%$ da área do território brasileiro e tornou-se importante na produção de grãos com a expansão das fronteiras agrícol as, com os L atossol os constituindo $46 \%$ dessa área (Resck, 1998).

A degradação do solo deve-se ao excessivo revolvimento da camada superficial pela utilização de sistemas de preparo convencional, com o uso de grades aradoras earados de discos. Tais sistemas de manejo, em geral, resultam na formação de camadas compactadas, levando ao aumento das perdas de solo, água e nutrientes e à redução da produtividade das culturas. Nos últimos anos, passou-se a questionar os sistemas de preparo intensivo adotados na região dos cerrados, buscando-secomo alternativa sistemas de manejo conservacionistas, entre os quais se destaca a semeadura direta, por revolver o solo apenas na linha de semeadura e promover menor alteração dos atributos físicos do solo, garantindo a sustentabilidade destes ao longo do tempo (Resck, 1998).

O uso intensivo de máquinas e implementos agrícolas na região do cerrado tem causado alterações químicas e físicas no solo em relação à condição de solo natural. Segundo Carvalho J r. et al. (1998) eSilva et al. (2000), Latossol os cultivados revelaram um aumento da argila dispersa em água e uma redução no grau de floculação do solo, favorecendo o aumento da densidade do solo e a formação de camadas compactadas.

Avaliações da estrutura do sol o podem ser feitas por meio de determinações indiretas que avaliam a quantidade deagregados estáveis em água (Oliveira et al., 1983, Silva et al., 1995; Silva et al., 2000).
Métodos que quantifiquem e qualifiquem as condições estruturais são importantes para avaliar a qualidade do solo (Carpenedo \& Mielniczuk, 1990), considerada um indicador da sustentabilidade dos sistemas de uso e manejo (Arshad et al., 1996; Harris et al., 1996; Muckel \& Mausbach, 1996).

Estudando agregados com vários graus de umidade, sob chuva simulada, Martinez-Mena et al. (1998) verificaram que o diâmetro médio geométrico dos referidos agregados apresentou correl ação positiva com o carbono orgânico $(r=0,49)$. Roth \& Haas (1989) e Campos et al. (1995) encontraram correlações positivas, $r=0,94$ e0,85, respectivamente, entre diâmetro médio geométrico dos agregados e matéria orgânica, em Latossolos do Paraná. Os efeitos da matéria orgânica na agregação estão relatados na literatura por vários autores, dentre os quais se destacam Tisdall \& Oades (1982); Roth et al. (1991); Hadas et al. (1994) e Campos et al. (1995).

Estudando sistemas de cultivo do solo, Castro Filho et al. (1998) verificaram que, na rotação de culturas milho/trigo/milho, a estabilidade de agregados foi $20 \%$ maior do que a da rotação soja/ trigo/soja, na profundidade de $0-10 \mathrm{~cm}$. Tais resultados foram atribuídos à maior produção de biomassa pel o milho, proporcionando mai or acúmulo de resíduos orgânicos que estimularam a atividade biológica, além do efeito do sistema radicular, resultando em maior estabilidade dos agregados. Efeitos positivos na agregação do solo propiciados por gramíneas foram observados também por Campos et al. (1995); Silva et al. (1998) e Silva \& Mielniczuk (1998).

Vários trabalhos, como os de Campos et al. (1995) e Castro Filho et al. (1998), têm mostrado maior estabilidade de agregados do solo em sistemas de 
semeadura direta, considerando a não-mobilização do sol oea deposição dematéria orgânica na superfície, incrementando a atividade biológica. Carpenedo \& Mielniczuk (1990), estudando a estabilidade estrutural dos agregados em condição natural e cultivada, verificaram redução da agregação quando os solos foram submetidos à aração e à gradagem para o cultivo de trigo e soja. Segundo Da Ros et al . (1997), o diâmetro médio geométrico dos agregados na semeadura direta, após cinco anos de cultivo, foi equivalente ao do campo nativo, diminuindo com o aumento da intensidade de preparo do solo, sendo 2,96 vezes maior do que no preparo convencional. Campos et al. (1995) constataram que, na semeadura direta, o diâmetro médio geométrico dos agregados foi superior ao do preparo convencional, cerca de duas vezes. Castro Filho et al. (1998) encontraram diâmetro médio geométrico $70 \%$ maior na semeadura direta do que no preparo convencional na camada superficial do solo.

A produtividade das culturas é resultante da interação de vários fatores, destacando-se os atributos do solo. Segundo Castro (1995), a produtividade média do milho foi de 5.634 e $5.687 \mathrm{~kg} \mathrm{ha}^{-1}$, na semeadura direta e no preparo convencional, respectivamente. Varios trabalhos têm mostrado pequenas diferenças de produtividade entre os sistemas de manejo (Pereira Filho \& Cruz, 1994; Santos et al., 1995).

O trabalho teve o objetivo deavaliar a agregação de um Latossolo Vermelho distrófico típico em diversos sistemas de manejo do solo na região dos cerrados em Sete Lagoas (MG).

\section{MATE RIAL E MÉTODOS}

O trabalho foi realizado na área experimental da Embrapa Milho e Sorgo, localizada em Sete Lagoas (MG), situada entre os paral el os $19^{\circ} 25^{\prime}$ sul e $44^{\circ} 15^{\prime}$ oeste, numa altitude de $732 \mathrm{~m}$, com temperatura e precipitação médias anuais de $22,1^{\circ} \mathrm{C}$ e $1.340 \mathrm{~mm}$, respectivamente. Segundo classificação de Köppen, o clima é Aw (tropical estacional de savana). O solo éum Latossolo Vermel ho distróficotípico A moderado textura muito argil osa fase cerrado tropical subcaducifólio, com relevo suave ondulado. Os atributos mineralógicos e químicos da camada superficial do solo estudado podem ser observados no quadro 1.

Os sistemas de manejo do sol o estudados foram: preparo convencional com grade aradora e cultivo contínuo com milho (CGCM); preparo convencional com arado de discos e cultivo contínuo com milho (CDCM ); preparo convencional com arado de discos e cultivo em rotação com milho e feijão (CDRMF); semeadura direta e cultivo contínuo com milho (SDCM ); semeadura direta ecultivo em rotação com milho e feijão (SDRMF) e cerrado nativo (CN) como testemunha.

\section{Quadro 1. Atributos mineralógicos e químicos em Latossolo Vermelho distrófico típico, na profundidade de 0-20 cm, Sete Lagoas (MG)}

\begin{tabular}{|c|c|c|c|c|c|c|c|}
\hline \multirow{2}{*}{ Ct } & \multirow{2}{*}{$\mathbf{G b}$} & \multicolumn{3}{|c|}{ Ataque sulfúrico } & \multirow{2}{*}{$\mathbf{K i}$} & \multirow{2}{*}{$\mathbf{K r}$} & \multirow{2}{*}{ PESN } \\
\hline & & $\mathrm{SiO}_{2}$ & $\mathrm{Al}_{2} \mathrm{O}_{3}$ & $\mathrm{Fe}_{2} \mathrm{O}_{3}$ & & & \\
\hline & 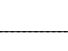 & $-\mathrm{g} \mathrm{kg}^{-1}$ & & - & & & \\
\hline 310 & 160 & 234 & 319 & 120 & 1,25 & 1,01 & 4,0 \\
\hline
\end{tabular}

Os cultivos em rotação de culturas vêm sendo efetuados desde 1992 e os cultivos sem rotação a partir de 1994. O milho foi semeado em outubro e, após a col heita, em abril, foi semeado o feijão em rotação. $\mathrm{O}$ experimento foi realizado sob condições irrigadas, utilizando o sistema de aspersão, sendo o controle da irrigação efetuado por baterias de tensiômetros instaladas a $20 \mathrm{~cm}$ de profundidade, irrigando-se sempre que a tensão de água no solo atingisse -0,7 bars. A produtividade do milho e do feijão foi obtida em área de $10 \mathrm{~m}^{2}$ por unidade experimental, sendo a umidade corrigi da para $15,5 \%$, para o milho, e 13\%, para o feijão.

F oram col etadas amostras de solo deformadas e indeformadas, em março de 1998, em três repetições, nas profundidades de 0-5, 5-20 e 20-30 cm, para determinações físicas e químicas em laboratório. Foram quantificados os teores de gibbsita (Gb) e caul inita (Ct) na fração argila desferrificada por meio da análise térmica diferencial eos óxidos $\left(\mathrm{SiO}_{2}, \mathrm{Al}_{2} \mathrm{O}_{3}\right.$ e $\mathrm{Fe}_{2} \mathrm{O}_{3}$ ) do ataque sulfúrico, na terra fina, segundo EMBRAPA (1997). Determinaram-se também os teores de carbono orgânico; a matéria orgânica foi obtida multiplicando-se estes teores pel o fator 1,724. A análise granulométrica do solo foi realizada pelo método de Bouyoucos (EMBRAPA, 1997), empregando-se $\mathrm{NaOH} 0,1 \mathrm{~mol} \mathrm{~L}^{-1}$ como dispersante químico na determinação da argila total e sem dispersante na determinação da argila dispersa em água, com agitação rápida (12.000 rpm), durante 20 min. Determinou-se também o índice de floculação(EMBRAPA, 1997).

Agregados com diâmetro entre 7,93-4,76 mm foram obtidos por meio de peneiramento de material indeformado do solo, e a estabilidade de agregados por meio do peneiramento em água, utilizando agregados sem pré-umedecimento e peneiras com abertura de malha de 4,76; 2,00; 1,00; 0,50; 0,25 e $0,105 \mathrm{~mm}$. Os resultados foram expressos em percentual dos agregados retidos nas peneiras $>2,0 \mathrm{~mm},<2,0 \mathrm{~mm}$, entre 2,0 e 1,0 $\mathrm{mm}$ e $<1,0 \mathrm{~mm}$ e diâmetro médio geométrico de acordo com Kemper \& Rosenau (1986). 
Aplicou-se análise da variância, segundo o delineamentoexperimental inteiramentecasualizado, com três repetições, considerando a profundidade de amostragem como subfator. Foi utilizado o teste de Scott \& Knott a 5\% para comparação entre as médias nos sistemas de manejo.

\section{RESULTADOSE DISCUSSÃO}

No quadro 2, estão apresentados os teores de matéria orgânica e alguns atributos físicos relacionados com a agregação do solo. Os maiores teores de matéria orgânica foram observados no cerrado nativo (CN) para todas as profundidades estudadas. Na profundidade de $0-5 \mathrm{~cm}$, os maiores teores de matéria orgânica foram observados nos sistemas com semeadura direta (SDCM e SDRMF), enquanto, na profundidade de $5-20 \mathrm{~cm}$, não foram observadas diferenças entre os sistemas de manejo. O menor teor de matéria orgânica observado no sistema de preparo convencional com grade aradora e cultivo contínuo com milho (CGCM), na profundidade de $20-30 \mathrm{~cm}$, deve-se, em parte, ao revolvimento mais superficial do solo propiciado pela grade aradora, com o arado de discos (CDCM e CDRMF ), ocasionando uma incorporação da matéria orgânica em maiores profundidades.

J á para a semeadura direta e cultivo continuo com milho(SDCM), o menor teor dematéria orgânica observado está relacionado com o pouco revol vimento do solo e, para a semeadura direta e cultivo com rotação com milho efeijão (SDRMF), na profundidade de 20-30 cm, esse teor não diferiu dos valores dos sistemas de manejo com arado de discos (CDCM e CDRMF), justificando o efeito benéfico da rotação de culturas, visto que, neste caso, os restos do sistema radicular do feijão incrementaram o teor de matéria orgânica. Efeitos positivos da matéria orgânica na agregação do solo foram observados por Tisdall \& Oades (1982); Roth et al. (1991), Hadas et al . (1994) e Silva et al. (1998).

O cerrado nativo (CN) apresentou, comotendência, os menores valores de argila dispersa em água e, conseqüentemente, os maiores valores de índice de floculação, em todas as profundidades estudadas (Quadro 2). Dentreos sistemas de manejo, noentanto, a semeadura direta com rotação de milho e feijão (SDRMF ) proporcionou os menores valores de argila dispersa em água e, conseqüentemente, os maiores val ores de índice de floculação, na camada de $0-5 \mathrm{~cm}$ (Carvalho J r. et al., 1998). Estes resultados estão coerentes com os mai ores teores de matéria orgânica (Quadro 2) na semeadura direta, quando comparados aos dos sistemas de manejo convencional. A matéria orgânica apresentou correlações significativas com o índice de floculação na camada de $0-5 \mathrm{~cm}$ do solo ( $r=0,89, P<0,01)$, comprovandoseu efeitona floculação das argilas, concordando com as observações de CarvalhoJ r. et al. (1998) eSilva et al. (2000).
Quadro 2. Atri butos químicos e físicos de Latossolo Vermelho distrófico típico, em diferentes profundidades e sistemas de manejo, Sete Lagoas (MG)

\begin{tabular}{|c|c|c|c|}
\hline Manejo & MO & ADA & IF \\
\hline & \multicolumn{2}{|c|}{$\mathrm{g} \mathrm{kg}^{-1} \longrightarrow$} & $\%$ \\
\hline \multicolumn{4}{|c|}{$0-5 \mathrm{~cm}$} \\
\hline CGCM & $36 c$ & $281 \mathrm{a}$ & $45,3 \mathrm{c}$ \\
\hline CDCM & $33 c$ & $298 a$ & $41,4 \mathrm{c}$ \\
\hline CDRMF & $38 c$ & $267 a$ & $46,8 \mathrm{c}$ \\
\hline SDCM & $44 \mathrm{~b}$ & $284 a$ & $41,7 \mathrm{c}$ \\
\hline SDRMF & $41 \mathrm{~b}$ & $228 \mathrm{~b}$ & $57,1 \mathrm{~b}$ \\
\hline $\mathrm{CN}$ & $52 a$ & $161 \mathrm{~b}$ & $72,0 \mathrm{a}$ \\
\hline \multicolumn{4}{|c|}{$5-20 \mathrm{~cm}$} \\
\hline CGCM & $37 \mathrm{~b}$ & $286 a$ & 46,5 a \\
\hline CDCM & $36 \mathrm{~b}$ & $254 a$ & 54,8 a \\
\hline CDRMF & $36 \mathrm{~b}$ & $245 a$ & $51,9 a$ \\
\hline SDCM & $36 \mathrm{~b}$ & 302 a & 43,1 a \\
\hline SDRMF & $33 \mathrm{~b}$ & $241 a$ & 56,4 a \\
\hline $\mathrm{CN}$ & $48 a$ & $179 \mathrm{~b}$ & $67,6 a$ \\
\hline \multicolumn{4}{|c|}{$20-30 \mathrm{~cm}$} \\
\hline $\mathrm{CGCM}$ & $24 \mathrm{~b}$ & $364 a$ & $36,9 \mathrm{~b}$ \\
\hline CDCM & $34 \mathrm{a}$ & $329 a$ & $41,5 \mathrm{~b}$ \\
\hline CDRMF & $32 a$ & $253 \mathrm{~b}$ & $53,7 a$ \\
\hline SDCM & $26 \mathrm{~b}$ & $334 a$ & $41,9 \mathrm{~b}$ \\
\hline SDRMF & $30 a$ & $288 \mathrm{~b}$ & $48,8 \mathrm{a}$ \\
\hline $\mathrm{CN}$ & $35 a$ & $241 \mathrm{~b}$ & $58,8 \mathrm{a}$ \\
\hline
\end{tabular}

MO: matéria orgânica; ADA: argila dispersa em água; IF : índice de floculação; Médias seguidas pela mesma letra, na coluna, e na mesma profundidade não diferem entre si pelo teste Scott e Knott $(P \leq 0,05)$.

Nos sistemas de manejo com semeadura direta (SDRMF e SDCM), com valores iguais de matéria orgânica (Quadro 2), o cultivo contínuo com milho (SDCM) propiciou menor valor de índice de floculação do solo na profundidade de $0-5 \mathrm{~cm}$. Esse fato pode ser atribuído à rotação de cultura com o feijão, alterando a qualidade da matéria orgânica pelo incremento de materiais orgânicos de rápida decomposi ção (baixa relação C:N ), oriundo dos restos culturais do feijão. I sso, provavelmente, acarretou liberação de maior quantidade de substâncias húmicas que atuam na agregação das partículas, decorrentes das alterações físico-químicas (Hadas et al., 1994; CarvalhoJ r. et al., 1998), sem, contudo, influenciar positivamente a agregação do solo. $\mathrm{Na}$ profundidade de $20-30 \mathrm{~cm}$, verificou-se efeito semelhante para o preparo convencional (CDCM), que proporcionou val ores inferiores para oíndice de floculação, com o mesmo teor de matéria orgânica do equivalente sistema de manejo com rotação (CDRMF). 
Tais tendências evidenciam que a qualidade e a cidagem da matéria orgânica atuam diferencialmente na floculação das argilas, indicando a necessidade demais estudos nesta linha de pesquisa Roth \& Haas (1989); Roth et al. (1991) e Castro Filho et al. (1998). Emestudo semelhante, Silva et al. (2000), investigando sistemas de manejo com sucessão trigo-soja em Latossolo Roxo, encontraram menor índice de floculação, na profundidade de $0-20 \mathrm{~cm}$, para o cultivo convencional com arado de discos comparado à semeadura direta.

$\mathrm{Na}$ figura 1, observam-se os resultados da estabilidade deagregados, expressos pela distribuição de agregados nas classes $>2,<2$, entre 2-1 e $<1$ $\mathrm{mm}$ para as profundidades estudadas. Na camada superficial $(0-5 \mathrm{~cm})$, as maiores percentagens de agregados $>2 \mathrm{~mm}$ foram observadas no cerrado nativo $(\mathrm{CN})$ e nos sistemas de manejo com semeadura direta (SDCM e SDRMF). Esses dados estão em sintonia com a correlação positiva e significativa entre a matéria orgânica e a percentagem de agregados $>2 \mathrm{~mm}$ (Quadro 3) e com o trabalho de Oliveira et al. (1983), os quais verificaram maiores valores dessa variável na classe de agregados $>2 \mathrm{~mm}$ com o incremento no teor de matéria orgânica do solo, concordando com Campos et al. (1995), Da Ros et al. (1997) e Castro Filho et al. (1998). I sto se deve, em parte, ao efeito mecânico do revolvimento do solo pelas operações de preparo convencional quefracionaram os agregados maiores em agregados menores, pela possível maior oxidação da matéria orgânica, indicada pelo seu menor teor nestes sistemas demanejo (Quadro 2), e pela possível redução dos microrganismos na agregação (Carpenedo \& Mielniczuk, 1990; Campos et al., 1995; Castro Filho et al., 1998). Na classe de 2-1 mm, não
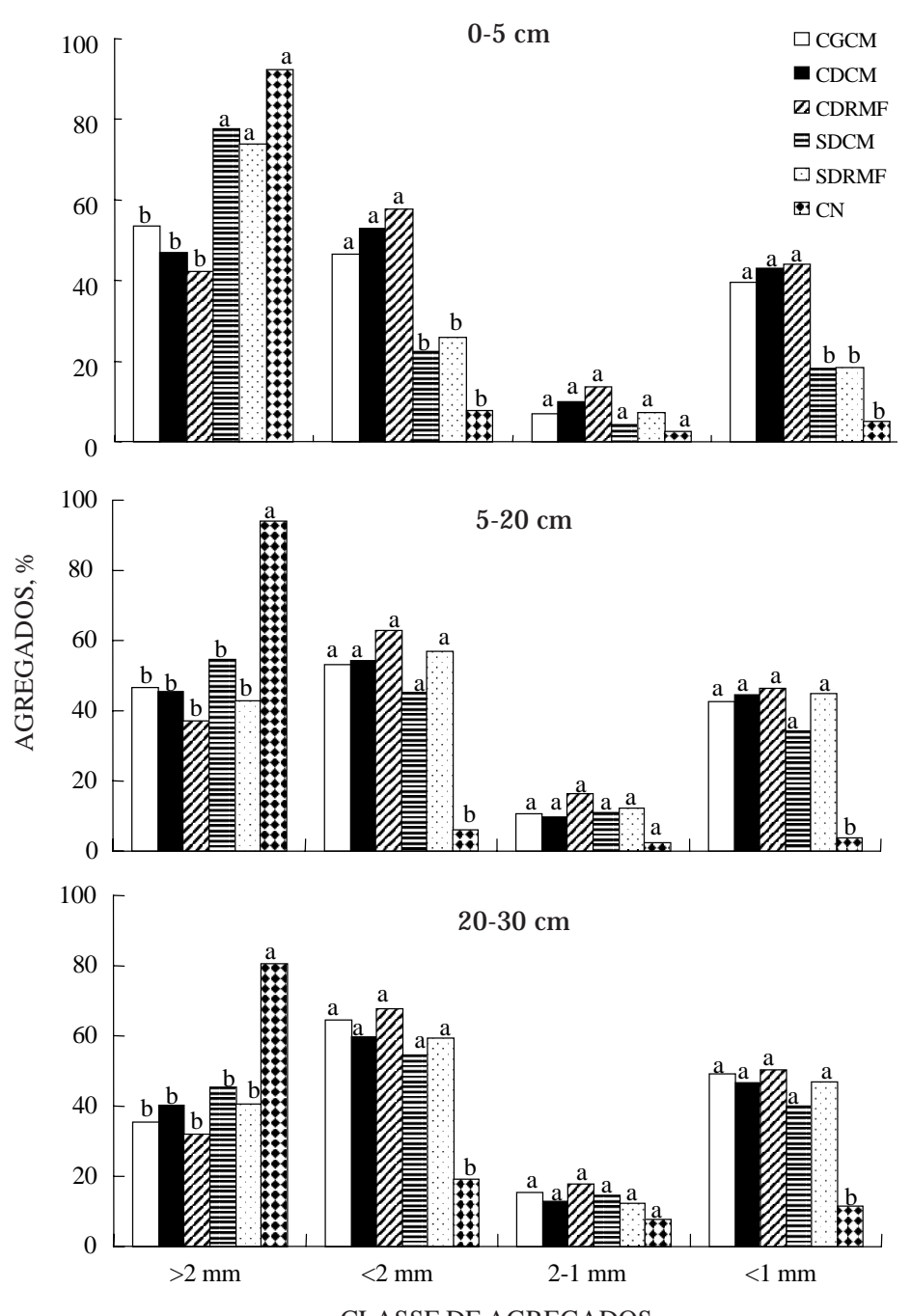

Figura 1. Distribuição do tamanho dos agregados estáveis em água em Latossolo Vermelho distrófico típico, em diferentes profundidades e sistemas de manejo, Sete Lagoas (MG). Letras acima das barras comparam sistemas de manejo, em diferentes classes de agregados, pelo teste Scott e Knott $(P \leq 0,05)$. 
Quadro 3. Coeficientes de correlação entre matéria orgânica eíndices de agregação em Latossolo Vermelho distrófico típico, em diferentes profundidades e sistemas de manejo, Sete Lagoas (MG)

\begin{tabular}{|c|c|c|c|}
\hline \multirow{3}{*}{ Atributo } & \multicolumn{3}{|c|}{ Coeficiente de correlação } \\
\hline & \multicolumn{3}{|c|}{ Profundidade $(\mathrm{cm})$} \\
\hline & 0-5 & 5-20 & 20-30 \\
\hline Matéria orgânica x Diâmetro médio geométrico (mm) & $0,97 * *$ & $0,05^{\text {ns }}$ & $0,47 \mathrm{~ns}$ \\
\hline Matéria orgânica x Agregados na classe > 2 mm (\%) & $0,92 * *$ & $-0,10^{\text {ns }}$ & $0,44^{\text {ns }}$ \\
\hline Matéria orgânica x Agregados na classe $<2$ mm (\%) & $-0,92 * *$ & $0,10^{\text {ns }}$ & $-0,44 \mathrm{~ns}$ \\
\hline Matéria orgânica x Agregados na classe 2 - 1 mm (\%) & $-0,74^{*}$ & $0,14^{\text {ns }}$ & $-0,48^{n s}$ \\
\hline Matéria orgânica $x$ Agregados na classe $<1 \mathrm{~mm}(\%)$ & $-0,93 * *$ & $0,08^{n s}$ & $-0,42^{n s}$ \\
\hline
\end{tabular}

*, ** e ns: significativos a 5 e 1\% e não-significativo, respectivamente.

foram observadas diferenças entre os sistemas de manejo. Nas camadas subsuperficiais (5-20 e 20$30 \mathrm{~cm}$ ) do solo, não foram observadas diferenças entre os sistemas de manejo, exceto entre o cerrado nativo (CN) e os sistemas de manejo estudados, nas classes $>2$, $<2$ e $<1 \mathrm{~mm}$, concordando com dados de Oliveira et al. (1983).

$\mathrm{Na}$ figura 2, observam-se os resultados da estabilidade de agregados, expressos pelo diâmetro médio geométrico (DMG). Os sistemas de manejo do solo propiciaram valores de diâmetro médio geométrico menor do que o do cerrado nativo (CN) em todas as profundidades (Figura 2). Isto permite inferir que todos os sistemas de manejo causaram efeitos negativos na estabilidade de agregados (Carpenedo\& Miel niczuk, 1990; Silva \& Mielniczuk, 1998). No cerrado nativo, foram encontrados os maiores valores de diâmetro médio geométrico em todas as profundidades os quais estão relacionados com os maiores teores de matéria orgânica, quetêm acentuada influência na agregação, e com a mel hor distribuição do sistema radicular das plantas de cerrado em profundidade no solo. Tais observações podem ser confirmadas pela correlação positiva e significativa encontrada entre diâmetro médio geométrico e matéria orgânica na camada supeficial (Quadro 3). Resultados semel hantes foram observados por Roth \& Haas (1989) e Castro Filho et al. (1998).

$\mathrm{Na}$ profundidade de $0-5 \mathrm{~cm}$, os sistemas de manejo convencional (CGCM, CDCM e CDPMF) apresentaram, em média, valores 1,86 e 2,85 vezes menores que os sistemas sob semeadura direta (SDCM eSDRMF) e cerrado nativo (CN), respectivamente. Esses val ores de diâmetro médio geométrico, superiores na semeadura direta comparados aos do preparo convencional, concordam com os estudos de Campos et al. (1995), Da Ros et al. (1997) e Castro Filho et al. (1998). Nas camadas subsuperficiais (5-20 e 20-30 cm), foram observados os efeitos mais pronunciados na redução do diâmetro médio geométrico dos agregados dos sistemas de manejo em relação ao cerrado nativo; os sistemas de manejo não diferiram entre si nestas profundidades (Figura 2). Nãoforam também observadas correlações significativas nas camadas subsuperficiais (Quadro 3), possivelmente por causa dos mais baixos teores de matéria orgânica no solo (Quadro 2). Essas observações concordam com as de Roth \& Haas (1989) e Campos et al. (1995).

O uso de indicadores físicos da qualidade do solo constitui importante estratégia para avaliar a sustentabilidade dos sistemas de manejo (Arshad et al ., 1996; Harris et al ., 1996; Muckel \& Mausbach, 1996). Neste estudo, os índices de estabilidade de agregados, notadamente a percentagem de agregados $<2,>2$ e $<1 \mathrm{~mm}$, e o diâmetro médio geométrico dos agregados apresentaram boa performance na distinção do efeito dos sistemas de manejo (Carpenedo \& Mielniczuk, 1990).

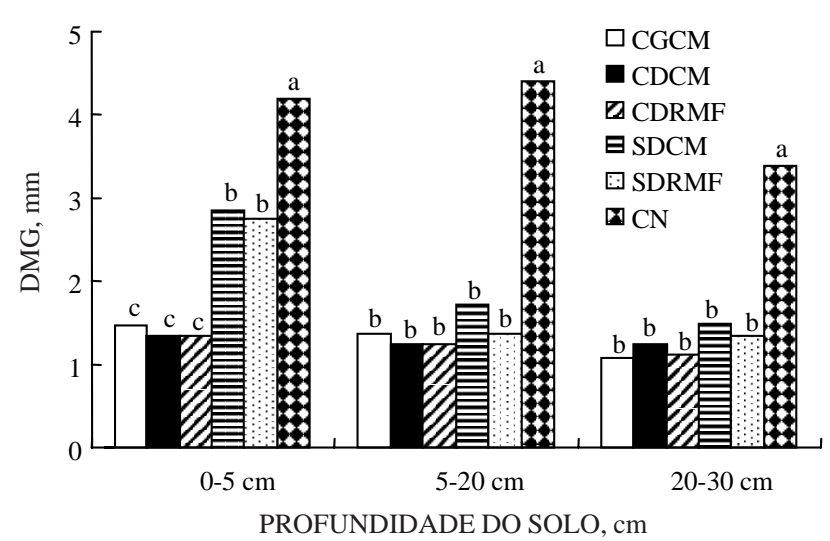

Figura 2. Diâmetro médio geométrico (DMG) dos agregados em Latossolo Vermelho distrófico típico, em diferentes profundidades e sistemas de manejo, Sete Lagoas (MG). Letras aci ma das barras comparam sistemas de manejo, em diferentes profundidades, pelo teste Scott e Knott $(P \leq 0,05)$. 
No quadro 4, observa-se a produtividade média das culturas do milho e do feijão para o período de estudo. A produtividade do milho nos sistemas sem rotação de cul turas mostrou val or inferior nosistema convencional com grade aradora (CGCM), enquanto a semeadura direta (SDCM) e osistema convencional com arado de discos (CDCM) proporcionaram produtividades semel hantes. Os sistemas com rotação de culturas (CDRMF e SDRMF) condicionaram maiores produtividades de milho. Já a produtividade média do feijão, para o período de estudo, foi superior na semeadura direta (SDRMF). Essas diferenças de produtividade entre sistemas demanejoforam observadas também por Pereira Filho \& Cruz (1994), Santos et al. (1995) e Castro (1995).

\section{Quadro 4. Produtividade média de milho (período de 1994 a 1998) e feijão (período de 1992 a 1996) em Latossolo Vermelho distrófico típico, em diferentes sistemas de manejo, Sete Lagoas (MG)}

\begin{tabular}{lcc}
\hline Manejo & Milho & Feijão \\
\cline { 2 - 3 } & & \multicolumn{2}{c}{$\mathrm{kg} \mathrm{ha}^{-1}$} & \\
CGCM & 4.875 & -- \\
CDCM & 5.112 & -- \\
CDRMF & 7.642 & 1.628 \\
SDCM & 5.114 & -- \\
SDRMF & 6.313 & 2.205 \\
\hline
\end{tabular}

\section{CONCLUSÃO}

1. A semeadura direta apresentou a maior percentagem de agregados na classe $>2 \mathrm{~mm}$ e as menores nas classes $<2 \mathrm{~mm}$ e $<1 \mathrm{~mm}$, bem como maior diâmetro médio geométrico dos agregados na superficie do solo. Não houve diferenças entre os sistemas de manejo para as camadas subsuperficiais.

\section{LITERATURA CITADA}

ARSHAD, M.A.; LOWERY, B. \& GROSSMAN, B. Physical tests for monitoring soil quality. I n: DORAN, J .W. \& J ONES, A.J ., eds. Methods for assessing soil quality. Madison, Soil Science Society of America, 1996. p.123-141 (SSSA Special Publication, 49)

CAMPOS, B.C.; REINERT, D.J .; NICOLODI, R.; RUEDELL, J . \& PETRERE, C. Estabilidade estrutural de um Latossolo Vermelho-Escuro distrófico após sete anos de rotação de culturas e sistemas de manejo de solo. R. Bras. Ci. Solo, 19:121-126, 1995.
CARPENEDO, V. \& MIELNICZUK, J . Estado de agregação e qualidade de agregados de Latossolos Roxos, submetidos a diferentes sistemas de manejo. R. Bras. Ci. Solo, 14:99-105, 1990.

CARVALHO Jr., I.A.; FONTES, L.E.F. \& COSTA, L.M. Modificações causadas pelo uso e a formação de camada compactada e, ou adensamento em Latossolo VermelhoEscuro textura média, na região dos cerrados. R. Bras. Ci. Solo, 22:505-514, 1998.

CASTRO FILHO, C.; MUZILLI, O. \& PODANOSCHI, A.L. Estabilidade dos agregados e sua relação com o teor de carbono orgânico num Latossolo Roxo distrófico, em função de sistemas de plantio, rotações de culturas e métodos de preparo das amostras. R. Bras. Ci. Solo, 22:527-538, 1998.

CASTRO, O.M. Comportamento físico e químico de um Latossolo Roxo em função do seu preparo na cultura do milho (Zea mays L.), Piracicaba, Escola Superior de Agricultura Luiz de Queiroz, 1995. 174p. (Tese de Doutorado)

DA ROS, C.O.; SECCO, D.; FIORIN, J .E.; PETRERE, C.; CADORE, M.A. \& PASA, L. Manejo do solo a partir de campo nativo: efeito sobre a forma e estabilidade da estrutura ao final de cinco anos. R. Bras. Ci. Solo, 21:241-247, 1997.

EMPRESA BRASILEIRA DE PESQUISA AGROPECUÁRIA EMBRAPA. Manual de métodos de análise de solo. 2.ed. Rio de J aneiro, Ministério da Agricultura, 1997. 212p.

HADAS, A.; RAWITZ, E.; ETKIN, H. \& MARGOLIN, M. Shortterm variations of soil physical properties as a function of the amount and $\mathrm{C} / \mathrm{N}$ ratio of decomposing cotton residues. I. Soil aggregation and aggregate tensilestrength. Soil Till. Res., 32:183-198, 1994.

HARRIS, R.F.; KARLEN, D.L. \& MULLA, D.J . A conceptual framework for assessment and management of soil quality and health. In: DORAN, J .W. \& J ONES, A.J ., eds. Methods for assessing soil quality. Madison, Soil Science Society of America, 1996. p. 61-82 (SSSA Special Publication, 49)

KEMPER, W.D. \& ROSENAU, R.C. Aggregate stability and size distribution. In: KLUTE, A., ed. Methods of soil analysis. 2.ed. Madison, American Society of Agromony, 1986. v.1. p.499-509.

MARTINEZ-MENA, M.; WILLIAMS, A.G.; TERNAN, J.L. \& FITZJ OHN, C. Role of antecedent soil water content on aggregates stability in a semi-arid environment. Soil Till. Res., 48:71-80, 1998.

MUCKEL, G.B. \& MAUSBACH, M.J . Soil quality information sheets. In: DORAN, J.W. \& J ONES, A.J ., eds. Methods for assessing soil quality. Madison, Soil Science Society of America, 1996. p.393-400 (SSSA Special Publication, 49)

OLIVEIRA, M.; CURI, N. \& FREIRE, J .C. Influência do cultivo na agregação de um Podzólico Vermelho-Amarelo textura média/argilosa da região de Lavras (MG). R. Bras. Ci. Solo, 7:317-322, 1983

PEREIRA FILHO, I.A.P. \& CRUZ, J.C. Efeitos da rotação de culturas e métodos de preparo de sol o na produção de milho e soja. In: RELATÓRIO TÉCNICO ANUAL DO CENTRO NACIONAL DE PESQUISA DE MILHO E SORGO 199293, 1994. p.180-181. 
RESCK, D.V.S. Plantio direto: desafios para os cerrados. In: REUNIÃO BRASILEIRA DE FERTILIDADE DO SOLO E NUTRIÇÃO DE PLANTAS, 23, REUNIÃO BRASILEIRA SOBRE MICORRIZAS, 7, SIMPÓSIO BRASILEIRO DE MICROBIOLOGIA DO SOLO, 5, REUNIÃO BRASILEIRA DE BIOLOGIA DO SOLO, 2, FERTIBIO 98, Caxambu, 1998. Resumos. Caxambu, 1998. p.32-33.

ROTH, C.H. \& HAAS, U. Efeito das interações fisico-químicas do sol o na agregação de um Latossol o Roxo. Berlin, Technische Universität Berlin, 1989. 20p. (Relatório Final)

ROTH, C.H.; CASTRO FILHO, C. \& MEDEIROS, G.B. Análise de fatores físicos e químicos relacionados com a agregação de um Latossolos Roxo distrófico. R. Bras. Ci. Solo, 15:241248, 1991.

SANTOS, H.P.; TOMM, G.O. \& LHAMBY, J .C.B. Plantio direto versus convencional: efeito na fertilidade do solo e no rendimento de grãos de culturas em rotação com cevada. R. Bras. Ci. Solo, 19:449-454, 1995
SILVA, I.F. \& MIELNICZUK, J. Sistemas de cultivo e características do solo afetando a estabilidade de agregados. R. Bras. Ci. Solo, 22:311-317, 1998

SILVA, M.L.N.; BLANCANEAUX, P.; CURI, N.; LIMA, J .M.; MARQUES, J .J.G.S.M. \& CARVALHO, A.M. Estabilidadee resistência de agregados de Latossolo Vermelho-Escuro cultivado com sucessão milho-adubo verde. Pesq. Agropec. Bras., 33:97-103, 1998.

SILVA, M.L.N.; CURI, N. \& BLANCANEAUX, P. Sistemas de manejo e qualidade estrutural de Latossolo Roxo. Pesq. Agropec. Bras., 35:2485-2492, 2000.

SILVA, M.L.N; CURI, N.; MARQUES, J JJ .G.S.M.; LIMA, L.A.; FERREIRA, M.M. \& LIMA, J .M. Resistência ao salpico provocado por impacto de gotas de chuva simulada em latossolos e sua relação com características químicas e mineralógicas. Ci. Prát., 19:182-188, 1995.

TISDALL, J .M. \& OADES, J .M. Organic matter and water-stable aggregates in soils. J. Soil Sci., 33:141-163, 1982. 